\title{
Dominant Secondary Nuclear Photoexcitation with the X-Ray Free-Electron Laser
}

\author{
Jonas Gunst, ${ }^{1, *}$ Yuri A. Litvinov, ${ }^{2}$ Christoph H. Keitel, ${ }^{1}$ and Adriana Pálffy ${ }^{1, \dagger}$ \\ ${ }^{1}$ Max-Planck-Institut für Kernphysik, Saupfercheckweg 1, D-69117 Heidelberg, Germany \\ ${ }^{2}$ GSI Helmholtzzentrum für Schwerionenforschung GmbH, 64291 Darmstadt, Germany
}

(Received 23 September 2013; revised manuscript received 8 December 2013; published 24 February 2014)

\begin{abstract}
The new regime of resonant nuclear photoexcitation rendered possible by x-ray free-electron laser beams interacting with solid state targets is investigated theoretically. Our results unexpectedly show that secondary processes coupling nuclei to the atomic shell in the created cold high-density plasma can dominate direct photoexcitation. As an example, we discuss the case of ${ }^{93 m}$ Mo isomer depletion for which nuclear excitation by electron capture as a secondary process is shown to be orders of magnitude more efficient than the direct laser-nucleus interaction. General arguments revisiting the role of the x-ray freeelectron laser in nuclear experiments involving solid-state targets are further deduced.
\end{abstract}

DOI: 10.1103/PhysRevLett.112.082501

23.20.Nx, 23.35.+g, 41.60.Cr, 52.25.Os

The new x-ray free-electron laser (XFEL) facilities [1,2] may provide both the $\mathrm{x}$-ray photon energies and the very high brilliance required for resonant driving of nuclear transitions [3-10]. So far, the resonant interaction between nuclei and the electromagnetic field has been studied in experiments performed with broadband synchrotron radiation (SR) [11] or bremsstrahlung [12]. The peak brilliance of XFEL light reaches up to 8 orders of magnitude higher than that of SR sources [13] and is expected to bring significant progress in light-nucleus interaction experiments. In particular, SR experiments with Mössbauer solid-state targets, mostly involving ${ }^{57} \mathrm{Fe}[14-16]$, provide only weak nuclear excitation despite the high target density and could benefit from the XFEL intensity. While so far in these experiments the electronic response only acted as background, the increase of the electric field strength leads to drastic changes in the interaction between photons and electrons which may additionally influence the nuclear excitation. Because of the unique interaction between high intensity $\mathrm{x}$-ray pulses and matter $[17,18]$, new states like cold, high-density plasmas can originate $[19,20]$. In such environments secondary nuclear processes from the coupling to the atomic shell are rendered possible by the presence of free electrons and atomic vacancies. This is also a new and diametrically opposed situation compared to photonuclear studies involving petawatt optical lasers [21-25].

In this Letter we investigate the nuclear excitation induced by the XFEL pulse shining on a nuclear solid-state target. We show that surprisingly, secondary nuclear excitation by electron capture (NEEC) in the occurring plasma can exceed by orders of magnitude the direct resonant photoexcitation despite the laser photons being tuned on the nuclear transition. Furthermore, we find that NEEC is more robust since it is less sensitive to the laser photon frequency fulfilling the resonance condition. This is a new feature as electronic processes were not relevant for experiments performed with $\mathrm{SR}$, where the fast electronic response of the sample was negotiated by time gating [26,27]. The concrete example studied here is the case of photoexcitation starting from the $6.85 \mathrm{~h}$ long-lived isomeric state of ${ }^{93} \mathrm{Mo}$ at approximately $2.5 \mathrm{MeV}$ excitation energy. The energy stored in the isomer may be released on demand by driving a $4.85 \mathrm{keV} E 2$ transition in an isomer triggering scenario [28-34]. Because of the advantageous energy ratio and available $\mathrm{x}$-ray light sources, isomer depletion in ${ }^{93} \mathrm{Mo}$ opens interesting prospects for the development of nuclear energy storage solutions. We show that for present XFEL parameters, the secondary nuclear excitation via NEEC is dominant and investigate under which conditions this is a general feature of the XFEL interaction with solid-state nuclear targets.

In the resonant process of NEEC, a free electron is captured into a bound atomic state by the simultaneous excitation of the nucleus [35] as illustrated in Fig. 1 for ${ }^{93 m}$ Mo. The nucleus is initially in its ground state or an excited metastable state, i.e., an isomer. The idea of isomer triggering is to connect the long-lived isomeric state with a higher level linked to freely radiating states as shown in

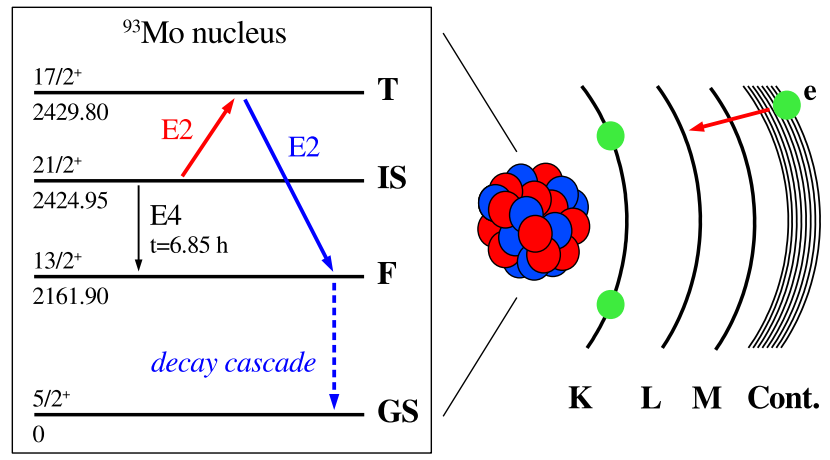

FIG. 1 (color online). $\quad{ }^{93 m}$ Mo excitation induced by NEEC into the $L$ shell (right) with subsequent decay to the nuclear ground state (long blue solid and dashed arrows in the left panel). The nuclear levels are labeled with their total angular momentum, parity, and energy (in keV). 
Fig. 1, in order to release the stored nuclear excitation energy on demand. In the case of ${ }^{93} \mathrm{Mo}$, a $4.85 \mathrm{keV}$ E2 triggering transition exists $[36,37]$ making this isomer particularly attractive for an XFEL-induced activation. Moreover, in ${ }_{41}^{93} \mathrm{Nb}(p, n){ }_{42}^{93 m}$ Mo reactions [38], the isomers can be produced directly embedded into $1 \mu \mathrm{m}$ thick solid-state niobium foils [39], providing high-density targets. Most of the XFEL photons will, however, interact with the atomic shells producing ionization and leading to plasma generation [18]. The study of NEEC in plasmas so far has been restricted to astrophysical environments [40-43] or optical-lasergenerated plasmas [44] where no equivalent of the direct photoexcitation channel under investigation here exists.

Currently, there are two operating XFEL facilities worldwide, the Linac Coherent Light Source (LCLS) at SLAC in Stanford, CA, USA, [1] and the SPring-8 Angstrom Compact free-electron laser (SACLA) in Japan [2], both capable of providing $4.85 \mathrm{keV}$ photons. The parameters of interest for our case are given in Table I. In addition, we also consider parameters for the European XFEL under construction at DESY in Hamburg, Germany [45]. The lightnucleus coupling can be described theoretically via the density matrix formalism using the semiclassical approach [46]. Typically, the nuclear decay happens on much slower time scales than the XFEL pulse duration, enabling us to restrict the interaction model to the two upper states in Fig. 1. As decoherent relaxation processes we include all spontaneous decay channels governed by radiative decay and internal conversion (IC), as well as a field-dephasing term that accounts for the limited coherence time. The large discrepancy between the laser width $\Gamma_{\text {XFEL }}(\sim 10 \mathrm{eV})$ and the nuclear transition width $\Gamma_{n}\left(\sim 10^{-7} \mathrm{eV}\right)$ allows only a small fraction of the laser photons to be resonant with the nuclear transition and justifies the introduction of effective laser parameters [6].

The dynamics of the nuclear occupation numbers is determined by the Bloch equations which can be written in terms of the interaction matrix elements $\left\langle T\left|H_{I}\right| \mathrm{IS}\right\rangle$, where $H_{I}$ represents the light-nucleus interaction, $|\mathrm{IS}\rangle$ the

TABLE I. The maximal achievable photon energy $E_{\max }$, bandwidth $\mathrm{BW}$, coherence time $T_{\text {coh }}$, peak intensity $I$, pulse repetition rate $f_{\text {rep }}$, and calculated triggering occupation number $\rho_{\text {trig }}$ after the photoexcitation of a single XFEL pulse for the three considered XFEL facilities.

\begin{tabular}{lccc}
\hline \hline & LCLS & SACLA & Eur. XFEL \\
Parameter & {$[47-49]$} & {$[50,51]$} & {$[45]$} \\
\hline$E_{\max }(\mathrm{eV})$ & 10332 & 19556 & 24800 \\
$\mathrm{BW}$ & $2 \times 10^{-3}$ & $2.2 \times 10^{-3}$ & $8 \times 10^{-4}$ \\
$T_{\text {coh }}(\mathrm{fs})$ & 2 & $\ldots$ a & 0.2 \\
$I\left(\mathrm{~W} / \mathrm{cm}^{2}\right)$ & $3.9 \times 10^{17}$ & $9.8 \times 10^{16}$ & $2.0 \times 10^{17}$ \\
$f_{\text {rep }}(\mathrm{Hz})$ & 30 & 10 & $4 \times 10^{4}$ \\
$\rho_{\text {trig }}$ & $1.8 \times 10^{-20}$ & $1.7 \times 10^{-20}$ & $2.4 \times 10^{-21}$ \\
\hline \hline
\end{tabular}

${ }^{\mathrm{a}}$ In our calculations we assumed $10 \%$ of the pulse duration, i.e., $10 \mathrm{fs}$. isomeric state and $|T\rangle$ the triggering level. This in turn can be related in the long-wavelength-approximation [52] to the reduced nuclear transition probabilities [36,37]. Following the formalism presented in Ref. [6], the Bloch equations are solved numerically. Results for the excited state occupation number $\rho_{\text {trig }}$, i.e., the fraction of excitation produced per laser pulse per nucleus in the sample, are shown in Table I. The calculations were performed considering a pulse duration of $100 \mathrm{fs}$ and a focal spot of $10 \mu \mathrm{m}^{2}$. The highest value $\rho_{\text {trig }}=1.8 \times 10^{-20}$ is achieved for the LCLS parameters.

A common problem of all presently operating XFELs is the poor temporal coherence (indicated by the coherence time $T_{\text {coh }}$ in Table I) due to random fluctuations in the initial electron charge density. The corresponding decoherence rate in the Bloch equations limits substantially the possible magnitude of $\rho_{\text {trig }}$. At the moment, there are two proposals on how to raise the temporal coherence of XFELs: (i) load the undulator with an already seeded light pulse in order to reduce shot-to-shot fluctuations at the start-up (seeded XFEL) [53-55]; (ii) construct an x-ray cavity based on diamond mirrors $[56,57]$ which directs the light pulse several times through the undulator [XFEL oscillator (XFELO)] [58]. Totally coherent $\mathrm{X}$-ray pulses increase the triggering occupation number by at least 4 and up to 6 orders of magnitude in comparison to their unseeded counterparts presented in Table I. Calculations with expected functioning parameters for the XFELO (with a pulse duration of $1 \mathrm{ps}$ ) deliver a value as high as $4.4 \times 10^{-14}$ for $\rho_{\text {trig }}$, proving the importance of the temporal coherence.

The nuclear excitation induced directly by the laser should be compared to its secondary electronic-processesinduced counterpart. The interaction of XFEL light with the electrons of the metallic target causes the direct production of inner shell holes, the uniform radiation of the sample and a rapid heating process [18], eventually leading to the formation of a plasma with unique properties, like uniform electron temperature and almost solid-state density [19]. In this environment, NEEC takes place on a longer time scale compared to the laser pulse duration, as long as suitable free electrons and atomic vacancies are available. In our estimate we consider this to hold for 100 ps after the laser pulse as long as the mass transport of the inner core plasma can be yet neglected [18].

The plasma evolution is modeled in two stages. First, plasma generation is dominated by the photoionization of inner shell electrons and the subsequent refilling of the arising holes by either radiative or Auger decays [20]. Note that in our scenario only holes in the $L$ shell and above can be produced by the laser photons due to the high $K$-shell ionization potentials. We estimate the laser energy deposited into the sample with the help of mass photoabsorption coefficients [59] which are in the first approximation held constant. By further accounting for energy conservation of the inner shell photoionization and the first sequence of Auger decays, the averaged electron temperature can be 
estimated [13]. As the next step following the XFELinduced plasma formation, radiative and collisional processes begin to dictate the further dynamics, and fast thermalization occurs. These are accounted for with the help of the population kinetics model implemented in the FLYCHK code [60]. The latter is based on a collisional radiative model and requires as input the averaged electron temperature $T_{e}$ and the ion density. In our case, we estimated $T_{e}=350 \mathrm{eV}$ for the $1 \mu \mathrm{m}$ thick Mo-Nb target and $10^{12}$ resonant laser photons with $10 \mu \mathrm{m}^{2}$ focus, and assumed the $\mathrm{Nb}$ solid-state density value. The electron density $n_{e}$ which adds up to $1.3 \times 10^{24} \mathrm{~cm}^{-3}$ and the charge state distribution (CSD) in the plasma are obtained using the FLYCHK code.

The total net NEEC rate in the plasma relies on the available charge states and electron energies. Performing a summation over the available charge states leads to

$$
\lambda_{\mathrm{NEEC}}=\sum_{q} P_{q} \lambda_{\mathrm{NEEC}}^{q}
$$

where $P_{q}$ denotes the probability to find an ion in the plasma with initial charge state $q$ before the capture. The partial NEEC rate $\lambda_{\text {NEEC }}^{q}$ contains a second summation over all contributing electronic capture levels $\left|\alpha_{d}\right\rangle$ and considers the available electron distribution $\phi_{e}(E)$ by a convolution integral over the electron kinetic energy $E$,

$$
\lambda_{\mathrm{NEEC}}^{q}=\sum_{\alpha_{d}} P_{\alpha_{d}} \int d E \sigma_{\mathrm{NEEC}}^{i \rightarrow d}(E) \varphi_{e}(E) .
$$

Here, $|i\rangle$ represents the initial state composed of the isomeric level $|\mathrm{IS}\rangle$ and the initial electronic state, and $|d\rangle$ the intermediate level determined by $|T\rangle$ and $\left|\alpha_{d}\right\rangle$. The probability to find $\left|\alpha_{d}\right\rangle$ unoccupied is denoted by $P_{\alpha_{d}}$. For a given charge state $q$ of the ion, we assume capture into free orbitals of the ion ground state only, i.e., in our case $P_{\alpha_{d}}=1$. Furthermore, in the derivation of Eq. (2) the single resonance approximation was applied, which allows us to write the total NEEC cross section in terms of the single resonances $\sigma_{\text {NEEC }}^{i \rightarrow d}$. The dependence of the latter on the freeelectron energy is given by [61] (in a.u.)

$$
\sigma_{\mathrm{NEEC}}^{i \rightarrow d}(E)=\frac{2 \pi^{2}}{p^{2}} Y_{\mathrm{NEEC}}^{i \rightarrow d} L_{d}\left(E-E_{d}\right),
$$

where $p$ is the momentum of the recombining electron, $Y_{\mathrm{NEEC}}^{i \rightarrow d}$ the NEEC transition width, and $L_{d}\left(E-E_{d}\right)$ the Lorentzian profile centered around the resonance energy $E_{d}$ with a width given by the natural width $\Gamma_{d}$ of the level $|d\rangle$. The momentum dependence implies that NEEC favors the capture into deep vacancies. The natural width of the resonance is given by the nuclear transition width if capture occurs into the electronic ground state, whereas otherwise also the width of the electronic bound initial state must be taken into account. The calculation of the NEEC width [61] involves the nuclear reduced transition probabilities and electronic matrix elements which we evaluate considering the single active electron approximation. The required many-electron wave functions, energies, and transition widths are obtained using the relativistic multiconfigurational Dirac-Fock method implemented in GRASP92 [62].

Results for the case of ${ }^{93 m}$ Mo triggering are shown in Fig. 2(a), where the single-resonance cross sections for several electron configurations undergoing NEEC into the $L, M, N$, and $O$ shells and the electron distributions for $T_{e}=350 \mathrm{eV}$ and $T_{e}=500 \mathrm{eV}$ are plotted as a function of the electron kinetic energy. Both the cross section and the number of available resonant electrons decrease with increasing $E$ or correspondingly by going to higher capture channels. Therefore, a cutoff level can be found for each charge state $q$ starting with which the NEEC excitation can be neglected. This procedure reduces the contributing resonances in Eq. (2) to a finite set of atomic levels, and provides a lower limit for $\lambda_{\text {NEEC }}$.

In Fig. 2(b) the partial NEEC rates $\lambda_{\mathrm{NEEC}}^{q}$ and the CSD are presented for $T_{e}=350 \mathrm{eV}$ and for comparison also for $T_{e}=500 \mathrm{eV}$ for charge states from $q=19+$ to $q=36+$. The excitation probability increases with $q$, since deeper
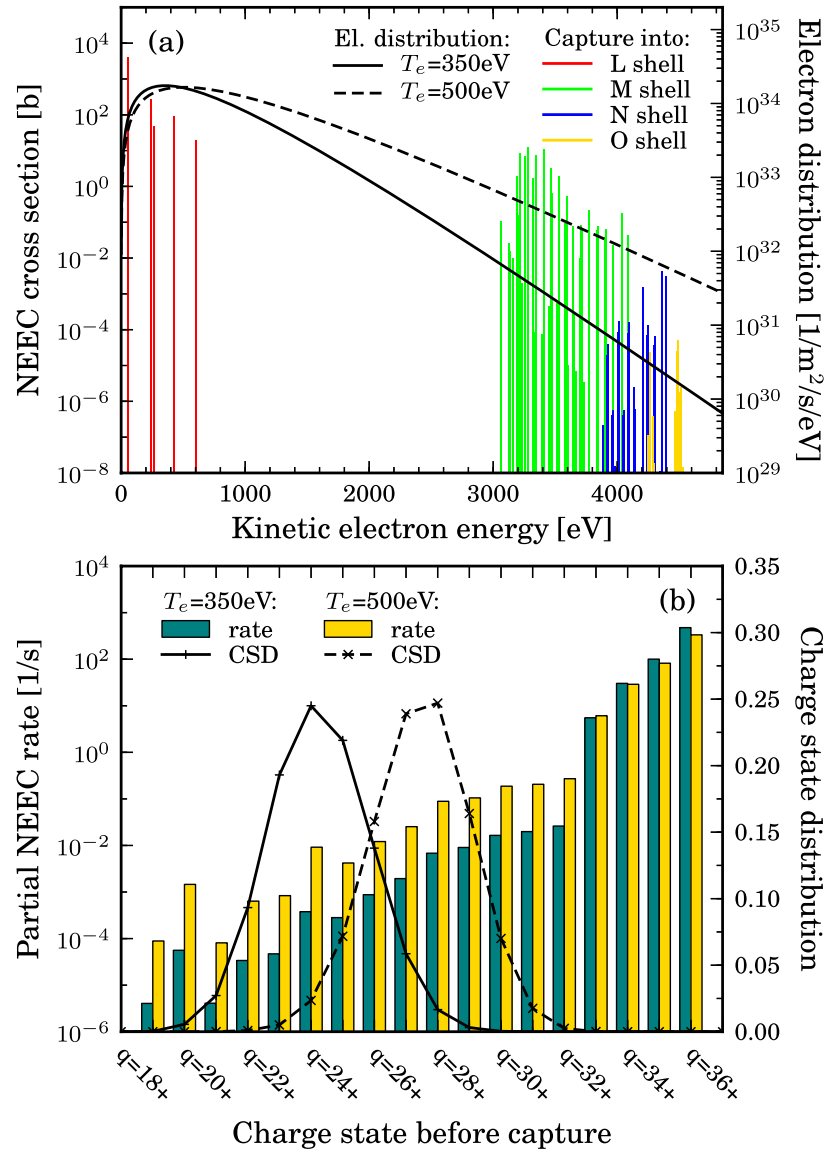

FIG. 2 (color online). (a) NEEC resonance cross sections $\sigma_{\text {NEEC }}$ for captures into the $L, M, N$, and $O$ shell (left axis) together with the electronic energy distribution (right axis). (b) Partial NEEC rate $\lambda_{\text {NEEC }}^{q}$ (left axis) together with the corresponding CSD (right axis). Results are presented for two plasma temperatures, $350 \mathrm{eV}$ and $500 \mathrm{eV}$. 
vacancies are present in higher charge states. Moreover, the CSD shows that only a limited number of charge states is available for a given plasma temperature $T_{e}$. For instance, in the case of $T_{e}=350 \mathrm{eV}$, NEEC into the $L$ shell is forbidden since this would require a Mo charge state of at least $q=33+$. Higher $q$ values can be reached by raising the plasma temperature, which can practically be implemented by turning the laser off resonance to higher frequencies [20]. With higher charge states available and an increase in the overall number of resonant electrons, the total NEEC rate $\lambda_{\text {NEEC }}$ increases with the plasma temperature $T_{e}$.

For $T_{e}=350 \mathrm{eV}$, the NEEC excitation entails a triggering occupation number of $\rho_{\text {trig }}=5.5 \times 10^{-14}$ per pulse which is about 6 orders of magnitude higher than the earlier indicated photoexcitation values for available XFEL parameters and is even competitive with the XFELO results. Already the comparison between the theoretical cross sections of NEEC and photoexcitation $[34,63]$ indicated that the coupling to the atomic shell can be more effective than to the radiation field for low-lying triggering levels. However, the main reason for the deviation between these two excitation mechanisms in our scenario is the discrepancy between the interaction times. While the photon-nucleus coupling is confined to the XFEL pulse duration ( $100 \mathrm{fs})$, the NEEC process takes place during the whole plasma lifetime which can be orders of magnitude longer. The total NEEC rates are also affected by other plasma parameters. Our estimates show a strong dependence of $\lambda_{\text {NEEC }}$ on the temperature $T_{e}$ and a weaker sensitivity towards the electron density $n_{e}$. For instance, a reduction of $10 \%$ in $T_{e}$ leads to a 4 times smaller NEEC rate, whereas a tenfold increase of the plasma volume results in a modification in $\lambda_{\text {NEEC }}$ of only $8 \%$. More extended, nonequilibrium calculations of the plasma parameters, especially for the electron temperature, could even open the way for using the NEEC excitation rate as a plasma diagnosis tool in future experiments.

Clearly measurable signatures for the nuclear excitation process are desirable for experiments. In the example of ${ }^{93 m}$ Mo triggering, an outstandingly high energetic photon of $1 \mathrm{MeV}$ is emitted in the decay cascade from the triggering level to the ground state regardless of the activation mechanism. Because of its high energy, such a $\gamma$-ray photon is unlikely to have electronic origin and can leave the plasma environment undisturbed. Although the emission may occur during the hydrodynamic expansion or even Coulomb explosion of the sample (lifetime of $|T\rangle$ is about $3 \mathrm{~ns}$ ) the preserved photonic energy of $1 \mathrm{MeV}$ can serve as a distinct signal for the triggering process.

Because of its resonant nature, the direct photoexcitation channel can be switched on and off by tuning the laser in or out of resonance, respectively. However, in the case of ${ }^{93} \mathrm{Mo}$, the $4.85 \mathrm{keV}$ triggering transition energy has at present an uncertainty of $80 \mathrm{eV}$ [36], which is a limiting factor for the experimental realization of direct photoexcitation given the eV XFEL bandwidth. In contrast, NEEC is not sensitive to the resonance condition because it takes place in a plasma environment where a broad electron energy distribution opens many resonance channels. Thus, not only is the NEEC triggering mechanism dominating over the direct channel, but it is also significantly more robust.

Considering the ${ }_{41}^{93} \mathrm{Nb}(p, n){ }_{42}^{93 m}$ Mo reaction cross section [38], a ${ }^{93 m}$ Mo isomer density of $10^{16} \mathrm{~cm}^{-3}$ can be achieved in the solid-state $\mathrm{Nb}$ foils [39] using standard proton beams like the LINAC at GSI $[64,65]$. For the resonant driving, assuming the European XFEL parameters, we obtain $9.6 \times 10^{-12}$ and $2.2 \times 10^{-4}$ signal photons/s induced by direct photoexcitation and NEEC, respectively, for a single $\mu \mathrm{m} \mathrm{Nb}$ target foil. Signal rates calculated for LCLS parameters are more than 2 orders of magnitude smaller, namely $5.6 \times 10^{-14}$ and $1.7 \times 10^{-7}$ signal photons/s, mainly due to the lower repetition rate. The XFEL pulse repetition can be exploited by using a tape-station system target. Moreover, it is possible to gain at least 1 order of magnitude in signal by using a stack of target foils as long as the laser intensity attenuation does not prohibit the plasma formation. Nevertheless, it would be desirable to increase the isomeric density in the laser focal spot, for instance by increasing the intensity or by a stronger focusing of the proton beam.

The generalization of our results for the excitation of other low-lying nuclear states (a suitable list is provided in Ref. [66]) via XFEL light relies on three aspects. First, the interaction time available for the plasma electrons to excite the nucleus depends on the hydrodynamic expansion time scale and can be orders of magnitude longer than the pulse duration over which resonant photons are present. Second, for the small excitation energies that can be reached with available and forthcoming XFEL machines ( $E \leq 25 \mathrm{keV})$, the NEEC cross sections are larger than the photoexcitation ones, as shown also by the large values $(\gg 1)$ of the IC coefficients [36], i.e., the ratios between the rates of the corresponding inverse processes IC and $\gamma$ decay. Finally, the XFEL photons resonant with the nuclear transition create in large numbers charge states and free electrons that are not far from the resonant NEEC condition. Only in the limit of high nuclear transition energies like for ${ }^{57} \mathrm{Fe}$ $(14.4 \mathrm{keV}),{ }^{149} \mathrm{Sm}(22.5 \mathrm{keV})$ or ${ }^{119} \mathrm{Sn}(23.9 \mathrm{keV})$, is the atomic photoabsorption cross section so strongly reduced that the NEEC resonance energies for the available charge states are expected rather at the falling high-energy tail of the electron distribution resulting in a low NEEC rate $\lambda_{\text {NEEC }}$. In all other cases listed in Ref. [66], inner shell vacancies characterized by high NEEC recombination cross sections will be produced which renders the successful capture of the generated low-energy plasma electrons possible. In addition, secondary nuclear excitation in the plasma is all the more to be considered in view of the typically high uncertainties of the nuclear transition energies which reduce from the start the chances for direct resonant photoexcitation.

We would like to thank D. Bauer for fruitful discussions. This research was in part supported by the Helmholtz-CAS Joint Research Group HCJRG-108. 
*Jonas.Gunst@mpi-hd.mpg.de

${ }^{\dagger}$ Palffy@mpi-hd.mpg.de

[1] Linac Coherent Light Source_LCLS (2013), http://lcls.slac .stanford.edu.

[2] SPring-8 Angstrom Compact Free Electron Laser-SACLA (2013), http://xfel.riken.jp/eng/sacla/.

[3] G. C. Baldwin and J. C. Solem, Rev. Mod. Phys. 69, 1085 (1997).

[4] H. Schwoerer, J. Magill, and B. Beleites, Lasers and Nuclei. Applications of Ultrahigh Intensity Lasers in Nuclear Science (Springer, Weinheim, 2006).

[5] T. J. Bürvenich, J. Evers, and C. H. Keitel, Phys. Rev. Lett. 96, 142501 (2006).

[6] A. Pálffy, J. Evers, and C. H. Keitel, Phys. Rev. C 77, 044602 (2008).

[7] I. Wong, A. Grigoriu, J. Roslund, T.-S. Ho, and H. Rabitz, Phys. Rev. A 84, 053429 (2011).

[8] R. N. Shakhmuratov, F. Vagizov, and O. Kocharovskaya, Phys. Rev. A 84, 043820 (2011).

[9] A. Di Piazza, C. Müller, K. Z. Hatsagortsyan, and C. H. Keitel, Rev. Mod. Phys. 84, 1177 (2012).

[10] B. W. Adams et al., J. Mod. Opt. 60, 2 (2013).

[11] Special Issue on "Nuclear Resonant Scattering of Synchrotron Radiation," edited by E. Gerdau and H. deWaard [Hyperfine Interact. 123-125, 1 (1999)].

[12] U. Kneissl, H. Pitz, and A. Zilges, Prog. Part. Nucl. Phys. 37, 349 (1996).

[13] M. Abd-Elmeguid et al., TESLA Technical Design Report. The X-Ray Free Electron Laser (DESY, Hamburg, 2001).

[14] Y. V. Shvyd'ko et al., Phys. Rev. Lett. 77, 3232 (1996).

[15] R. Röhlsberger, K. Schlage, B. Sahoo, S. Couet, and R. Rüffer, Science 328, 1248 (2010).

[16] R. Röhlsberger, H.-C. Wille, K. Schlage, and B. Sahoo, Nature (London) 482, 199 (2012).

[17] L. Young et al., Nature (London) 466, 56 (2010).

[18] S. P. Hau-Riege, High-Intensity X-rays-Interaction with Matter (WILEY-VCH, Weinheim, 2011).

[19] R. W. Lee et al., J. Opt. Soc. Am. B 20, 770 (2003).

[20] S. M. Vinko et al., Nature (London) 482, 59 (2012).

[21] K. W. D. Ledingham et al., Phys. Rev. Lett. 84, 899 (2000).

[22] T. E. Cowan et al., Phys. Rev. Lett. 84, 903 (2000).

[23] P. Gibbon, Short Pulse Laser Interactions with Matter. An Introduction (Imperial College Press, London, 2005).

[24] K. M. Spohr et al., New J. Phys. 10, 043037 (2008).

[25] G. Mourou and T. Tajima, Science 331, 41 (2011).

[26] R. Röhlsberger, Nuclear Condensed Matter Physics With Synchrotron Radiation: Basic Principles, Methodology and Applications (Springer-Verlag, Berlin, 2004).

[27] W. Sturhahn, J. Phys. Condens. Matter 16, S497 (2004).

[28] P. Walker and G. Dracoulis, Nature (London) 399, 35 (1999).

[29] A. Aprahamian and Y. Sun, Nat. Phys. 1, 81 (2005).

[30] D. Belic et al., Phys. Rev. Lett. 83, 5242 (1999).

[31] C. B. Collins et al., Phys. Rev. Lett. 82, 695 (1999).

[32] D. Belic et al., Phys. Rev. C 65, 035801 (2002).

[33] J. J. Carroll, Laser Phys. Lett. 1, 275 (2004).

[34] A. Pálffy, J. Evers, and C. H. Keitel, Phys. Rev. Lett. 99, 172502 (2007).

[35] A. Pálffy, Contemp. Phys. 51, 471 (2010).

[36] Nuclear Structure and Decay Databases (2013), http://www .nndc.bnl.gov/.
[37] M. Hasegawa, Y. Sun, S. Tazaki, K. Kaneko, and T. Mizusaki, Phys. Lett. B 696, 197 (2011).

[38] Experimental Nuclear Reaction Data-EXFOR (2013), http://www-nds.iaea.org/exfor/exfor.htm/.

[39] See Supplemental Material at http://link.aps.org/ supplemental/10.1103/PhysRevLett.112.082501 for ISOMER PRODUCTION (2013).

[40] G. Gosselin and P. Morel, Phys. Rev. C 70, 064603 (2004).

[41] P. Morel, V. Méot, G. Gosselin, D. Gogny, and W. Younes, Phys. Rev. A 69, 063414 (2004).

[42] G. Gosselin, V. Méot, and P. Morel, Phys. Rev. C 76, 044611 (2007).

[43] P. Morel, V. Méot, G. Gosselin, G. Faussurier, and C. Blancard, Phys. Rev. C 81, 034609 (2010).

[44] M. R. Harston and J. F. Chemin, Phys. Rev. C 59, 2462 (1999).

[45] M. Altarelli et al., The European X-Ray Free Electron Laser. Technical Design Report (DESY, Hamburg, 2007).

[46] M. O. Scully and M.S. Zubairy, Quantum Optics (Cambridge University, New York, 2008).

[47] J. Arthur et al., Linac Coherent Light Source (LCLS). Conceptual Design Report (SLAC, Stanford, 2002).

[48] P. Emma et al., Nat. Photonics 4, 641 (2010).

[49] C. Gutt et al., Phys. Rev. Lett. 108, 024801 (2012).

[50] S. Goto et al., XFEL/SPring-8. Beam Line Technical Design Report (RIKEN, Japan, 2010).

[51] T. Ishikawa et al., Nat. Photonics 6, 540 (2012).

[52] P. Ring and P. Schuck, The Nuclear Many-Body Problem (Springer, New York, 1980).

[53] J. Feldhaus, E. Saldin, J. Schneider, E. Schneidmiller, and M. Yurkov, Opt. Commun. 140, 341 (1997).

[54] E. Saldin, E. Schneidmiller, Y. Shvyd'ko, and M. Yurkov, Nucl. Instrum. Methods Phys. Res., Sect. A 475, 357 (2001).

[55] G. Geloni, V. Kocharyan, and E. Saldin, Extension of SelfSeeding to Hard X-Rays $>10 \mathrm{keV}$ as a Way to Increase User Access at the European XFEL (DESY, Hamburg, 2011).

[56] Y. V. Shvyd'ko, S. Stoupin, A. Cunsolo, A. H. Said, and X. Huang, Nat. Phys. 6, 196 (2010).

[57] Y. Shvyd'ko, S. Stoupin, V. Blank, and S. Terentyev, Nat. Photonics 5, 539 (2011).

[58] K.-J. Kim, Y. Shvyd'ko, and S. Reiche, Phys. Rev. Lett. 100, 244802 (2008).

[59] B. Henke, E. Gullikson, and J. Davis, At. Data Nucl. Data Tables 54, 181 (1993).

[60] H.-K. Chung, M. Chen, W. Morgan, Y. Ralchenko, and R. Lee, High Energy Density Phys. 1, 3 (2005).

[61] A. Pálffy, W. Scheid, and Z. Harman, Phys. Rev. A 73, 012715 (2006).

[62] K. Dyall, I. Grant, C. Johnson, F. Parpia, and E. Plummer, Comput. Phys. Commun. 55, 425 (1989).

[63] A. Pálffy, J. Mod. Opt. 55, 2603 (2008).

[64] Gesellschaft für Schwerionenforschung GSI Darmstadt (2013), http://www.gsi.de.

[65] Proton Linac for FAIR at GSI (2013), https://www.gsi .de/fs2/C/start/beschleuniger/fachabteilungen/linac/projects/ proton_linac_for_fair.htm.

[66] A. Junker, A. Pálffy, and C. H. Keitel, New J. Phys. 14, 085025 (2012). 